October 1, 2018

\title{
FROM PRINCIPAL CHIRAL MODEL TO SELF-DUAL GRAVITY
}

\author{
Jerzy F. Plebański, ${ }^{\star}$ Maciej Przanowski ${ }^{\dagger}$ \\ And Hugo García-Compeán ${ }^{\ddagger}$ \\ Departamento de Física \\ Centro de Investigación y de Estudios Avanzados del IPN. \\ Apdo. Postal 14-740, 07000, México D.F., México.
}

\begin{abstract}
It is demonstrated that the action of $\mathrm{SU}(N)$ principal chiral model leads in the limit $N \rightarrow \infty$ to the action for Husain's heavenly equation. The principal chiral model in the Hilbert space $L^{2}\left(\Re^{1}\right)$ is considered and it is shown, that in this case the chiral equation is equivalent to the Moyal deformation of Husain's heavenly equation. New method of searching for solutions to this latter equation, via Lie algebra representations in $L^{2}\left(\Re^{1}\right)$ is given.
\end{abstract}

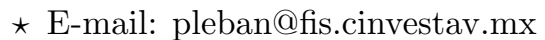

$\dagger$ Permanent address: Institute of Physics, Technical University of Lódź, Wólczańska 219, 93-005 Lódź, Poland.

$\ddagger$ E-mail : compean@fis.cinvestav.mx
} 


\section{Introduction}

Recently a great deal of interest has been devoted to the relation between chiral models and self-dual gravity ${ }^{1-4}$. In particular, Husain ${ }^{3}$ has shown that the Ashtekar-Jacobson-Smolin equations describing the self-dual vacuum metric can be written a $\mathrm{s}$ the principal chiral model equations for the Lie algebra $\operatorname{sdiff}\left(\Sigma^{2}\right)$. This enables him to derive a new form of the self-dual gravity equation which we call Husain's heavenly equation.

In the present paper we show that Husain's heavenly equation can be derived from the action which appears to be the $N \rightarrow \infty$ limit of the action for the $\mathrm{SU}(N)$ principal chiral model (Section 2).

Then in Section 3 we consider the principal chiral model in the Hilbert space $L^{2}\left(\Re^{1}\right)$. We prove that in this case the chiral equation is in fact the Moyal deformation of Husain's heavenly equation.

Finally, in Section 4 it is shown that given a solution of the principal chiral equations for some complex Lie algebra $\mathcal{G}_{C}$ and a representation of $\mathcal{G}_{C}$ into the Lie

algebra $\hat{\mathcal{I}}$ of linear operators acting in $L^{2}\left(\Re^{1}\right)$ one get s, via the Weyl-Wigner-Moyal formalism the solution of the Moyal deformation of Husain's heavenly equation. Then, assuming that this solution is analytic with respect to the deformation parameter $\hbar$ and putting $\hbar \rightarrow 0$ one finds the solution of Husain's heavenly equation.

Therefore, in a sense, the looking for the self-dual vacuum metrics consists in the searching for the Lie algebra representations in the Hilbert space $L^{2}\left(\Re^{1}\right)$.

Two natural questions arise (a)-. What is the connection between our method and the twistor method?

(b)-. Does the method presented, provide us with a model of the well known, but still mysterious, procedure ${ }^{5-7} \operatorname{su}(N \rightarrow \infty) \cong \operatorname{sdiff}\left(\Sigma^{2}\right)$ ?

We intend to consider these problems elsewhere. 


\section{The principal chiral model approach to self-dual gravity}

We deal with G-principal chiral model in a simply connected open submanifold $\mathcal{V}$ of the two-dimensional Euclidean space $\Re^{2}$ of Cartesian coordinates $(x, y)$. Here $\mathbf{G}$ stands for a matrix real Lie group. The real Lie algebra of $\mathbf{G}$

will be denoted by $\mathcal{G}$.

The principal chiral equations can be written as follows

$$
\begin{gathered}
\partial_{x} A_{y}-\partial_{y} A_{x}+\left[A_{x}, A_{y}\right]=0 \\
\partial_{x} A_{x}+\partial_{y} A_{y}=0
\end{gathered}
$$

where $A_{\mu} \in \mathcal{G} \otimes C^{\infty}(\mathcal{V}), \mu \in\{x, y\}$, are the chiral potentials.

From (2.1a) one infers that $A_{x}$ and $A_{y}$ are of the pure gauge form i.e.,

$$
A_{\mu}=g^{-1} \partial_{\mu} g
$$

where $g=g(x, y) \in \mathbf{G}, \mu \in\{x, y\}$.

Substituting (2.2) into (2.1b) we get the well known principal chiral equations

$$
\partial_{x}\left(g^{-1} \partial_{x} g\right)+\partial_{y}\left(g^{-1} \partial_{y} g\right)=0
$$

However, another approach is also available ${ }^{8}$. Namely, from Eq. (2.1b) it follows that

$$
A_{x}=-\partial_{y} \theta, \quad \text { and } \quad A_{y}=\partial_{x} \theta
$$

where $\theta=\theta(x, y)$ is some $\mathcal{G}$-valued $C^{\infty}$-real function on $\mathcal{V}$, i.e, $\theta \in \mathcal{G} \otimes C^{\infty}(\mathcal{V})$. Inserting then (2.4) into (2.1a) one obtains the principal chiral equations in the followin $\mathrm{g}$ form 


$$
\partial_{x}^{2} \theta+\partial_{y}^{2} \theta+\left[\partial_{x} \theta, \partial_{y} \theta\right]=0
$$

Assume that the Lie algebra $\mathcal{G}$ of $\mathbf{G}$ is semisimple. Then by straightforward calculations one can show that Eqs. (2.5) are the result of the variational principle ${ }^{8}$

$$
\begin{gathered}
\delta S_{C h}=0, \quad S_{C h}=\int_{\mathcal{V}} \mathcal{L}_{C h} d x d y \\
\mathcal{L}_{C h}:=\alpha \operatorname{Tr}\left\{\frac{1}{3} \theta\left[\partial_{x} \theta, \partial_{y} \theta\right]-\frac{1}{2}\left(\left(\partial_{x} \theta\right)^{2}+\left(\partial_{y} \theta\right)^{2}\right)\right\} .
\end{gathered}
$$

where $\alpha>0$ is some constant. (Compare also with Ref.9).

Consider now the case when

$$
\mathbf{G}=\operatorname{SDiff}\left(\Sigma^{2}\right)
$$

where $\Sigma^{2}$ is a real two-dimensional flat symplectic manifold of the symplectic form $\omega=d q \wedge d p$. Then in the case considered we have ${ }^{5-7}$

$$
\mathcal{G}=\operatorname{sdiff}\left(\Sigma^{2}\right) \cong \text { the Poisson bracket algebra on } \Sigma^{2}
$$

Consequently, $\theta \in \operatorname{sdiff}\left(\Sigma^{2}\right) \otimes C^{\infty}(\mathcal{V})$ is a Hamiltonian vector field

$$
\theta=\frac{\partial \Theta}{\partial q} \frac{\partial}{\partial p}-\frac{\partial \Theta}{\partial q} \frac{\partial}{\partial p}
$$

where $\Theta=\Theta(x, y, p, q)$. Substituting (2.9) into (2.5) one gets the following equation 


$$
\partial_{x}^{2} \Theta+\partial_{y}^{2} \Theta+\left\{\partial_{x} \Theta, \partial_{y} \Theta\right\}_{P}=\mathcal{Y}
$$

where $\mathcal{Y}=\mathcal{Y}(x, y)$ is some real function and $\{\cdot, \cdot\}_{P}$ stands for the Poisson bracket on $\Sigma^{2}$, i.e.,

$$
\left\{\partial_{x} \Theta, \partial_{y} \Theta\right\}_{P}:=\frac{\partial\left(\partial_{x} \Theta\right)}{\partial q} \frac{\partial\left(\partial_{y} \Theta\right)}{\partial p}-\frac{\partial\left(\partial_{x} \Theta\right)}{\partial p} \frac{\partial\left(\partial_{y} \Theta\right)}{\partial q}
$$

From the very definition of $\Theta(2.9)$ one quickly infers that without any loss of generality the function $\mathcal{Y}$ can be chosen to vanish. Finally we arrive at the $\operatorname{sdiff}\left(\Sigma^{2}\right)$ - principal chiral equation

$$
\partial_{x}^{2} \Theta+\partial_{y}^{2} \Theta+\left\{\partial_{x} \Theta, \partial_{y} \Theta\right\}_{P}=0
$$

This is exactly Husain's heavenly equation ${ }^{3}$ determining the self-dual metric on $\mathcal{V} \times \Sigma^{2}$. (For other heavenly equations see Refs. 9,10 and 11). The evident formal similarity between Eqs. (2.5) and Eq. (2.12) may suggest that this latter equation can be derived from the variational principle analogous to (2.6). Indeed, by simple computations one finds that Eq. (2.12) appears to be the Euler-Lagran ge equation for the following variational problem

$$
\begin{gathered}
\delta S_{G}=0, \quad S_{G}=\int_{\mathcal{V} \times \Sigma^{2}} \mathcal{L}_{G} d x d y d p d q \\
\mathcal{L}_{G}:=-\frac{1}{3} \Theta\left\{\partial_{x} \Theta, \partial_{y} \Theta\right\}_{P}+\frac{1}{2}\left(\left(\partial_{x} \Theta\right)^{2}+\left(\partial_{y} \Theta\right)^{2}\right) .
\end{gathered}
$$

(In the cases of the first or second heavenly equations ${ }^{9}$ the Lagrangians analogous to $\mathcal{L}_{G}$ have been considered by Boyer, Finley and one of us (J.F.P.) ${ }^{10}$. Compare also $\mathcal{L}_{G}$ with Ref. 9). 
Note that the variational principle (2.13) can be justified as follows: Let $\mathbf{G}=$ $\mathrm{SU}(\mathrm{N})$ and, consequently, $\mathcal{G}=\mathrm{su}(\mathrm{N})$. It is well known that

$$
\operatorname{su}(\infty) \cong \operatorname{sdiff}\left(\Sigma^{2}\right)
$$

Then, taking the constant $\alpha$ in (2.6) to be now

$$
\alpha=\frac{(2 \pi)^{4}}{N^{3}}
$$

one can prove that $S_{G}$ appears to be an $N \rightarrow \infty$ limit of $S_{C h}$. This limit can be obtained formally by the substitutions ${ }^{9}$

$$
\begin{aligned}
\frac{(2 \pi)^{4}}{N^{3}} \operatorname{Tr}(\cdots) & \rightarrow-\int_{\Sigma^{2}}(\cdots) d p d q, \\
\theta & \rightarrow \Theta \\
{\left[\partial_{x} \theta, \partial_{y} \theta\right] } & \rightarrow\left\{\partial_{x} \Theta, \partial_{y} \Theta\right\}_{P},
\end{aligned}
$$

(About $\mathrm{su}(N \rightarrow \infty)$ see Refs. 5,6,7 and 9).

\section{The principal chiral model in a Hilbert space and the Moyal deformation of the heavenly equation}

Here we consider the case when the group $\mathbf{G}$ appears to be the group of unitary operators acting in the Hilbert space $L^{2}\left(\Re^{1}\right)$. Then, $\mathcal{G}$ is now the Lie algebra of the anti-self-dual operators in $L^{2}\left(\Re^{1}\right)$. To make our notation more tra nsparent

we use in the present case the symbols $\hat{\mathbf{U}}$ and $\hat{\mathcal{U}}$ for $\mathbf{G}$ and $\mathcal{G}$, respectively. The principal chiral equations (2.5) take now the form of the operator equation 


$$
\partial_{x}^{2} \hat{\theta}+\partial_{y}^{2} \hat{\theta}+\left[\partial_{x} \hat{\theta}, \partial_{y} \hat{\theta}\right]=0
$$

$$
\hat{\theta} \in \hat{\mathcal{U}} \otimes C^{\infty}(\mathcal{V})
$$

Finally, defining the self-dual operator-valued function $\hat{\Theta}=\hat{\Theta}(x, y)$

$$
\hat{\Theta}:=i \hbar \hat{\theta}, \quad \hat{\Theta} \in \mathcal{O} \otimes C^{\infty}(\mathcal{V})
$$

where $\mathcal{O}$ stands for the set of the self-dual operators in $L^{2}\left(\Re^{1}\right)$, we get the principal chiral equation in the Hilbert space $L^{2}\left(\Re^{1}\right)$ to be

$$
\partial_{x}^{2} \hat{\Theta}+\partial_{y}^{2} \hat{\Theta}+\frac{1}{i \hbar}\left[\partial_{x} \hat{\Theta}, \partial_{y} \hat{\Theta}\right]=0
$$

By simple calculations one can quickly show that Eq. (3.3) can be derived from the following variational principle (compare with Ref. 9)

$$
\begin{gathered}
\delta S_{C h}^{(q)}=0, \quad S_{C h}^{(q)}=\int_{\mathcal{V}} \mathcal{L}_{C h}^{(q)} d x d y \\
\mathcal{L}_{C h}^{(q)}:=\operatorname{Tr}\left\{2 \pi \hbar\left[-\frac{1}{3 i \hbar} \hat{\Theta}\left[\partial_{x} \hat{\Theta}, \partial_{y} \hat{\Theta}\right]+\frac{1}{2}\left(\left(\partial_{x} \hat{\Theta}\right)^{2}+\left(\partial_{y} \hat{\Theta}\right)^{2}\right)\right]\right\} \\
=2 \pi \hbar \sum_{j}<\psi_{j}\left|\left\{-\frac{1}{3 i \hbar} \hat{\Theta}\left[\partial_{x} \hat{\Theta}, \partial_{y} \hat{\Theta}\right]+\frac{1}{2}\left(\left(\partial_{x} \hat{\Theta}\right)^{2}+\left(\partial_{y} \hat{\Theta}\right)^{2}\right)\right\}\right| \psi_{j}>
\end{gathered}
$$

where $\left\{\mid \psi_{j}>\right\}_{j \in \mathcal{N}}$ is the orthonormal basis in $L^{2}\left(\Re^{1}\right)$ 


$$
<\psi_{j}\left|\psi_{k}>=\delta_{j k}, \quad \sum_{j}\right| \psi_{j}><\psi_{j} \mid=\hat{I} .
$$

Now we arrive at the point where the Wigner-Weyl-Moyal formalism ${ }^{12-18,9}$ can be applied. By the Weyl correspondence one gets the real function on $\mathcal{V} \times \Re^{2}$ defined as follows

$$
\Theta=\Theta(x, y, p, q):=\int_{-\infty}^{+\infty}<q-\frac{\xi}{2}|\hat{\Theta}| q+\frac{\xi}{2}>\exp \left(\frac{i p \xi}{\hbar}\right) d \xi .
$$

As $\hat{\Theta}$ satisfies Eq. (3.3) the function $\Theta$ satisfies the equation

$$
\partial_{x}^{2} \Theta+\partial_{y}^{2} \Theta+\left\{\partial_{x} \Theta, \partial_{y} \Theta\right\}_{M}=0,
$$

where $\{\cdot, \cdot\}_{M}$ stands for the Moyal bracket i.e.,

$$
\left\{f_{1}, f_{2}\right\}_{M}:=\frac{1}{i \hbar}\left(f_{1} * f_{2}-f_{2} * f_{1}\right)=\frac{2}{\hbar} f_{1} \sin \left(\frac{\hbar}{2} \stackrel{\leftrightarrow}{\mathcal{P}}\right) f_{2},
$$

where $\overleftrightarrow{\mathcal{P}}: \frac{\overleftarrow{\partial}}{\partial q} \frac{\vec{\partial}}{\partial p}-\frac{\overleftarrow{\partial}}{\partial p} \frac{\vec{\partial}}{\partial q}, f_{1}=f_{1}(x, y, p, q)$

$f_{2}=f_{2}(x, y, p, q)$, with the Moyal $*$-product defined by

$$
f_{1} * f_{2}:=f_{1} \exp \left(\frac{i \hbar}{2} \stackrel{\leftrightarrow}{\mathcal{P}}\right) f_{2}
$$

From (3.8) and (3.9) one easily infers that

$$
\lim _{\hbar \rightarrow 0} f_{1} * f_{2}=f_{1} f_{2} \text { and } \lim _{\hbar \rightarrow 0}\left\{f_{1}, f_{2}\right\}_{M}=\left\{f_{1}, f_{2}\right\}_{P} .
$$

Therefore, Eq. (3.7) is evidently the Moyal deformation of Husain's heavenly equation (2.12). 
One is also able to express $S_{C h}^{(q)}$ and $\mathcal{L}_{C h}^{(q)}$ in terms of $\Theta$ defined by (3.6). A straightforward computation shows that

$$
\begin{gathered}
S_{C h}^{(q)}=\int_{\mathcal{V} \times \Re^{2}} \mathcal{L}_{G}^{(M)} d x d y d p d q \\
\mathcal{L}_{C h}^{(q)}=\int_{\Re^{2}} \mathcal{L}_{G}^{(M)} d p d q \\
\mathcal{L}_{G}^{(M)}:=-\frac{1}{3} \Theta *\left\{\partial_{x} \Theta, \partial_{y} \Theta\right\}_{M}+\frac{1}{2}\left(\left(\partial_{x} \Theta\right) *\left(\partial_{x} \Theta\right)+\left(\partial_{y} \Theta\right) *\left(\partial_{y} \Theta\right)\right) .
\end{gathered}
$$

Thus $\mathcal{L}_{G}^{(M)}$ can be considered to be the Lagrangian for Eq. (3.7). Of course, by (3.10) one has

$$
\lim _{\hbar \rightarrow 0} \mathcal{L}_{G}^{(M)}=\mathcal{L}_{G}
$$

It is of some interest to consider in the present case the principal chiral equations as written in the usual form (2.3).

We now have evidently

$$
\partial_{x}\left(\hat{g}^{-1} \partial_{x} \hat{g}\right)+\partial_{y}\left(\hat{g}^{-1} \partial_{y} \hat{g}\right)=0
$$

where $\hat{g}=\hat{g}(x, y)$ is an $\hat{\mathbf{U}}$-valued function on $\mathcal{V}$. Eq. (3.13) can be easily found to follow from the variational principle 


$$
\begin{aligned}
& \delta S_{C h}^{\prime(q)}=0, \quad S_{C h}^{(q)}=\int_{\mathcal{V}} \mathcal{L}_{C h}^{(q)} d x d y \\
& \mathcal{L}_{C h}^{\prime(q)}=-\pi \hbar^{3} \operatorname{Tr}\left\{\left(\hat{g}^{-1} \partial_{\mu} \hat{g}\right)\left(\hat{g}^{-1} \partial_{\mu} \hat{g}\right)\right\} \\
& =\pi \hbar^{3} \operatorname{Tr}\left\{\left(\partial_{\mu} \hat{g}\right)\left(\partial_{\mu} \hat{g}^{-1}\right)\right\}, \quad \mu \in\{x, y\}
\end{aligned}
$$

(Summation over $\mu$ !). The Lagrangian (3.14) is well known in the principal chiral model theory ${ }^{20}$. The constant $\pi \hbar^{3}$ is chosen for further convenience.

Using the Weyl-Wigner-Moyal formalism one gets

$$
\begin{gathered}
S_{C h}^{\prime(q)}=\int_{\mathcal{V} \times \Re^{2}} \mathcal{L}_{G}^{(M)} d x d y d p d q \\
\mathcal{L}_{C h}^{(q)}=\int_{\Re^{2}} \mathcal{L}_{G}^{\prime(M)} d p d q=\int_{\Re^{2}} \mathcal{L}_{G}^{\prime \prime(M)} d p d q, \\
\mathcal{L}_{G}^{(M)}:=-\frac{\hbar^{2}}{2}\left(g^{-1} * \partial_{\mu} g\right) *\left(g^{-1} * \partial_{\mu} g\right), \\
\mathcal{L}_{G}^{\prime \prime(M)}:=\frac{\hbar^{2}}{2}\left(\partial_{\mu} g\right) *\left(\partial_{\mu} g^{-1}\right),
\end{gathered}
$$

where

$$
g=g(x, y, p, q):=\int_{-\infty}^{+\infty}<q-\frac{\xi}{2}|\hat{g}| q+\frac{\xi}{2}>\exp \left(\frac{i p \xi}{h}\right) d \xi
$$

and $g^{-1}$ is the Moyal *-product inverse of $g$ i.e., 


$$
g^{-1} * g=g * g^{-1}=1 .
$$

Moreover, as $\hat{g}^{\dagger}=\hat{g}^{-1}$ one has

$$
g^{-1}=\bar{g}
$$

where the bar stands for the complex conjugation.

In terms of the function $g=g(x, y, p, q)$ Eq. (3.13) reads

$$
\partial_{\mu}\left(g^{-1} * \partial_{\mu} g\right)=0
$$

This last equation is equivalent to the Moyal deformation of Husain's heavenly equation (3.7) the correspondence between Eq. (3.7) and Eq. (3.20) is given by

$$
g^{-1} * \partial_{x} g=-\frac{1}{i \hbar} \partial_{y} \Theta, \quad g^{-1} * \partial_{y} g=\frac{1}{i \hbar} \partial_{x} \Theta
$$

In terms of $\Theta$ the Lagrangian $\mathcal{L}_{G}^{\prime(M)}$ reads

$$
\mathcal{L}_{G}^{\prime(M)}=\frac{1}{2}\left[\left(\partial_{x} \Theta\right) *\left(\partial_{x} \Theta\right)+\left(\partial_{y} \Theta\right) *\left(\partial_{y} \Theta\right)\right]
$$

The difference between $\mathcal{L}_{G}^{(M)}$ defined by $(3.11)$ and $\mathcal{L}_{G}^{\prime(M)}$

$$
\mathcal{L}_{G}^{(M)}-\mathcal{L}_{G}^{(M)}=-\frac{1}{3} \Theta *\left\{\partial_{x} \Theta, \partial_{y} \Theta\right\}_{M}
$$

when the relations (3.21) are applied, vanishes. Indeed, one quickly shows that 


$$
\left\{\partial_{x} \Theta, \partial_{y} \Theta\right\}_{M}=\left\{g^{-1} * \partial_{y} g, g^{-1^{*}} * \partial_{x} g\right\}_{M} \equiv 0 .
$$

Gothering all that, one concludes that Eq. (3.20) with (3.18) give an equivalent to (3.7) description of the Moyal deformation of Husain's heavenly equation.

Finally, we make an important remark. The considerations of Sects. 2 and 3 can be easily generalized on the case of the complex Lie algebras $\mathcal{G}_{C}$. In this case

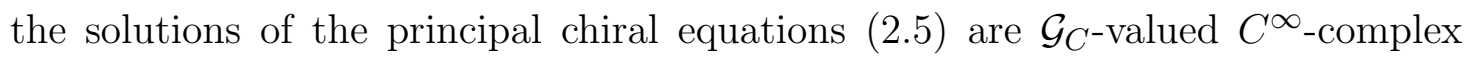
functions i.e. $\theta=\theta(x, y) \in \mathcal{G}_{C} \otimes C^{\infty}(\mathcal{V} ; \mathbf{C})$ and the solutions of Husain's equation (2.12) or of its Moyal deformation (3.7) are admitted to be complex. Therefore, consequently, instead of $\hat{\mathcal{U}}$ one deals with the complex Lie algebra of operators in $L^{2}\left(\Re^{1}\right)$. This algebra will be denoted by $\hat{\mathcal{I}}$. Thus we have now $\hat{\Theta} \in \hat{\mathcal{I}} \otimes C^{\infty}(\mathcal{V} ; \mathbf{C})$ and the function $\Theta=\Theta(x, y, p, q)$ defined by (3.6) is, in general, a complex function. Then the extension of the results on the case of complex coordinates $(x, y, p, q)$ is automatic.

\section{Remarks on looking for the solutions}

The results of the last two sections provide us with a promising method for searching for the solutions of Husain's heavenly equation (2.12).

Indeed, let

$$
\Phi: \mathcal{G}_{C} \rightarrow \hat{\mathcal{I}}
$$

be a Lie algebra homomorphism. This implies that if

$$
\theta=\theta(x, y)=\theta_{a}(x, y) \tau_{a} \in \mathcal{G}_{C} \otimes C^{\infty}(\mathcal{V} ; \mathbf{C})
$$

where $\tau_{a} \in \mathcal{G}_{C}, a=1, \ldots, \operatorname{dim} \mathcal{G}_{C}$, constitute the basis of $\mathcal{G}_{C}$, is a solution of the principal chiral equations (2.5), then 


$$
\begin{gathered}
\hat{\Theta}=\hat{\Theta}(x, y)=i \hbar \theta_{a} \hat{X}_{a} \in \hat{\mathcal{I}} \otimes C^{\infty}(\mathcal{V} ; \mathbf{C}) \\
\hat{X}_{a}:=\Phi\left(\tau_{a}\right)
\end{gathered}
$$

satisfies Eq. (3.3). (Summation over $a$ is assumed!). Consequently, the function

$$
\Theta=\Theta(x, y, p, q)=i \hbar \theta_{a}(x, y) X_{a}(p, q)
$$

where $($ see $(3.6))$

$$
X_{a}=X_{a}(p, q):=\int_{-\infty}^{+\infty}<q-\frac{\xi}{2}\left|\hat{X}_{a}\right| q+\frac{\xi}{2}>\exp \left(\frac{i p \xi}{\hbar}\right) d \xi
$$

appears to be a solution of Eq. (3.7). Assume then, that the function $\Theta$ is an analytic function of $\hbar$ i.e.,

$$
\begin{gathered}
\Theta=\sum_{n=0}^{\infty} \hbar^{n} \Theta_{n}, \\
\Theta_{n}=\Theta_{n}(x, y, p, q) .
\end{gathered}
$$

As $\Theta$ is a solution of $(3.7)$ and $\lim _{\hbar \rightarrow 0}\{\cdot, \cdot\}_{M}=\{\cdot, \cdot\}_{P}$ the function $\Theta_{0}$ fulfills Husain's heavenly equation (2.12).

It is evident from our previous considerations (see especially the end of $\S 3$ ) that if one restricts oneself to the real Lie algebra $\mathcal{G}$ and, then, to the homomorphism

$$
\Phi: \mathcal{G} \rightarrow \hat{\mathcal{U}}
$$

then one arrives at the real solution $\Theta_{0}$. 
Finally, the self-dual metric is defined $b y^{3}$

$d s^{2}=d x\left(\Lambda_{x p} d p+\Lambda_{x q} d q\right)+d y\left(\Lambda_{y p} d p+\Lambda_{y q} d q\right)+\frac{1}{\left\{\Lambda_{x}, \Lambda_{y}\right\}_{P}}\left[\left(\Lambda_{x p} d p+\Lambda_{x q} d q\right)^{2}+\left(\Lambda_{y p} d p+\Lambda_{y y} d q\right)^{2}\right]$

$$
\Lambda \equiv \Theta_{0}
$$

where $\Lambda_{x p} \equiv \partial_{x} \partial_{p} \Lambda, \Lambda_{y p} \equiv \partial_{y} \partial_{p} \Lambda, \ldots$ etc.

\subsection{EXAMPLES}

$\underline{\text { The SU(2) Chiral Model }}$

Here, the solution of the principal chiral equations (2.5) can be written in the form

$$
\begin{gathered}
\theta=\theta(x, y)=\theta_{a}(x, y) \tau_{a}, \quad a=1,2,3 \\
\tau_{1}=\frac{i}{2}\left(\begin{array}{cc}
0 & 1 \\
1 & 0
\end{array}\right), \quad \tau_{2}=\frac{i}{2}\left(\begin{array}{cc}
0 & -i \\
i & 0
\end{array}\right), \quad \tau_{3}=\frac{i}{2}\left(\begin{array}{cc}
1 & 0 \\
0 & -1
\end{array}\right), \\
{\left[\tau_{a}, \tau_{b}\right]=\epsilon_{a b c} \tau_{c} .}
\end{gathered}
$$

We define the Lie algebra homomorphism $\Phi: \mathrm{su}(2) \rightarrow \hat{\mathcal{I}}$ by $^{20}$ 


$$
\begin{gathered}
\Phi\left(\tau_{1}\right)=\hat{X}_{1}:=i \beta \hat{q}+\frac{1}{2 \hbar}\left(\hat{q}^{2}-1\right) \hat{p} \\
\Phi\left(\tau_{2}\right)=\hat{X}_{2}:=-\beta \hat{q}+\frac{i}{2 \hbar}\left(\hat{q}^{2}+1\right) \hat{p} \\
\Phi\left(\tau_{3}\right)=\hat{X}_{3}:=-i \beta \hat{1}-\frac{1}{\hbar} \hat{q} \hat{p}
\end{gathered}
$$

where $\beta \in \Re$ is any constant; $\hat{q}$ and $\hat{p}=-i \hbar \frac{\partial}{\partial q}$ are the position and momentum operators, respectively. Then, the complex function $\Theta=\Theta(x, y, p, q)$ defined by (4.4) and (4.5) is a solution of Eq.(3.7) i.e., of the Moyal deformation of Husain's heavenly equation.

Inserting (4.10) into (4.5) and, then the result into (4.4) one quickly finds $\Theta$ to be of the form

$$
\Theta=\frac{i}{2} \theta_{1} p\left(q^{2}-1\right)-\frac{1}{2} \theta_{2} p\left(q^{2}+1\right)-i \theta_{3} p q+\hbar \cdot\left(\beta+\frac{1}{2}\right) \cdot\left(-\theta_{1} q-i \theta_{2} q+\theta_{3}\right) .
$$

Consequently, the function $\Theta_{0}$

$$
\Theta_{0}=\frac{i}{2} \theta_{1} p\left(q^{2}-1\right)-\frac{1}{2} \theta_{2} p\left(q^{2}+1\right)-i \theta_{3} p q
$$

appears to be a complex solution of Husain's heavenly equation (2.12). It is evident, that taking

$$
\beta=-\frac{1}{2}+\frac{\gamma}{\hbar}, \quad \gamma \in \Re,
$$

one finds another solution of Eq. (2.12); namely

$$
\Theta_{0}^{\prime}=\Theta_{0}+\gamma \cdot\left(-\theta_{1} q-i \theta_{2} q+\theta_{3}\right)
$$


The $S L(2 ; \Re)$ Chiral Model

Now the solution of the principal chiral equations (2.5) takes the form

$$
\begin{gathered}
\theta=\theta(x, y)=\theta_{a}(x, y) \tau_{a}, \quad a=1,2,3, \\
\tau_{1}=\frac{1}{2}\left(\begin{array}{cc}
0 & 1 \\
1 & 0
\end{array}\right), \quad \tau_{2}=\frac{1}{2}\left(\begin{array}{cc}
0 & -1 \\
1 & 0
\end{array}\right), \quad \tau_{3}=\frac{1}{2}\left(\begin{array}{cc}
1 & 0 \\
0 & -1
\end{array}\right), \\
{\left[\tau_{1}, \tau_{2}\right]=\tau_{3}, \quad\left[\tau_{2}, \tau_{3}\right]=\tau_{1}, \quad\left[\tau_{3}, \tau_{1}\right]=-\tau_{2} .}
\end{gathered}
$$

Following Ref. 21 we define the Lie algebra homomorphism $\Phi: \operatorname{sl}(2 ; \Re) \rightarrow \hat{\mathcal{U}}$ by

$$
\begin{gathered}
\Phi\left(\tau_{1}\right)=\hat{X}_{1}:=\frac{i}{4}\left(\frac{\hat{p}^{2}}{\hbar^{2}}+\frac{\delta}{\hat{q}^{2}}-\hat{q}^{2}\right), \\
\Phi\left(\tau_{2}\right)=\hat{X}_{2}:=\frac{i}{4}\left(\frac{\hat{p}^{2}}{\hbar^{2}}+\frac{\delta}{\hat{q}^{2}}+\hat{q}^{2}\right), \\
\Phi\left(\tau_{3}\right)=\hat{X}_{3}:=\frac{i}{2}\left(\frac{\hat{q} \hat{p}}{\hbar}-\frac{i}{2}\right),
\end{gathered}
$$

where $\delta \in \Re$ is a constant.

Defining the functions $X_{a}=X_{a}(p, q)$ according to (4.5) and inserting them into (4.4) we get the real solution of the Moyal deformation of Husain's equation 
to be

$\Theta=\Theta(x, y, p, q)=\hbar^{-1}\left[-\frac{1}{4}\left(\theta_{1}+\theta_{2}\right) p^{2}\right]-\frac{1}{2} \theta_{3} p q+\hbar\left[-\frac{\delta}{4}\left(\theta_{1}+\theta_{2}\right) q^{-2}+\frac{1}{4}\left(\theta_{1}-\theta_{2}\right) q^{2}\right]$

Thus one arrives at the solution which is not an analytic function in $\hbar$ if $\theta_{1}+\theta_{2} \neq 0$. Therefore, in general, the function

$$
\Theta_{0}=-\frac{1}{2} \theta_{3} p q
$$

doesn't satisfy Eq. (2.12).

Assume

$$
\theta_{1}+\theta_{2}=0
$$

Then, using the results of Ref. 20 one shows that $\theta_{3}=\theta_{3}(x, y)$ is of the following form

$$
\partial_{x} \theta_{3}=\partial_{y} F, \quad \partial_{y} \theta_{3}=-\partial_{x} F
$$

where $F=F(x, y)$ is an arbitrary real function.

Consequently, $\Theta_{0}$ defined by (4.18) and (4.20) fulfills Husain's heavenly equation (2.12) but, as now $\left\{\partial_{x} \Theta_{0}, \partial_{y} \Theta_{0}\right\}_{P}=0$, this solution doesn't define any selfdual metric (see (4.8)).

\section{Acknowledgments}

We are indebted to T. Matos for useful discussions on the principal chiral model. One of us (M.P.) would like to thank the staff of the Department of Physics at CINVESTAV, México D.F., México, for the warm hospitality. This work is supported by CONACyT and CINVESTAV, México, D.F., México. 


\section{References}

1. Q-Han Park, Int. J. Mod. Phys. A7, 1415 (1992).

2. I.A.B. Strachan, Phys. Lett. B283, 63 (1992).

3. V. Husain, Phys. Rev. Lett. 72, 800 (1994); Class. Quantum Grav. 11, 927 (1994).

4. H. García-Compeán and T. Matos, Solutions in self-dual gravity constructed via chiral equations, to appear in Phys. Rev. D52 (1995).

5. J. Hoppe, Phys. Lett. B215, 706 (1988).

6. D.B. Fairlie, P. Fletcher and C.K. Zachos, J. Math. Phys. 31, 1088 (1990).

7. E.G. Floratos, J. Iliopoulos and G. Tiktopoulos, Phys. Lett. B217, 285 (1989).

8. C.R. Nappi, Phys. Rev. D21, 418 (1980).

9. J.F. Plebański and M. Przanowski, The Lagrangian of self-dual gravitational field as a limit of the SDYM Lagrangian, submitted to Phys. Lett. A.

10. J.F. Plebański, J. Math. Phys. 16, 2395 (1975).

11. C.P. Boyer, J.D. Finley III and J.F. Plebański, Complex relativity, $\mathcal{H}$ and $\mathcal{H} \mathcal{H}$ spaces- a survey, in General Relativity and Gravitation, Einstein memorial volume, vol.2, ed. A. Held (Plenum, New York, 1980) pp. 241-281.

12. H. Weyl, Z. Phys. 46, 1 (1927).

13. E.P. Wigner, Phys. Rev. 40, 749 (1932).

14. J.E. Moyal, Proc. Cambridge Phil. Soc. 45, 99 (1949).

15. J.F. Plebański, Institute of Physics of Nicolaus Copernicus University, Toruń, preprint No. 69 (1969).

16. F. Bayen, M. Flato, C. Fronsdal, A. Lichnerowicz and D. Sternheimer, Ann. Phys. NY 111, 61 (1978); Ann. Phys. NY 111, 111 (1978). 
17. M. Hillery, R.F. O'Connell, M.O. Scully and E.P. Wigner, Phys. Rep. 106, 121 (1984).

18. W.I. Tatarskij, Usp. Fiz. Nauk 139, 587 (1983).

19. N. Sanchez, Exact solutions in gauge theory, general relativity, and their supersymmetric extensions, in Geometric Aspects of the Einstein Equations and Integrable Systems, ed. R. Martini (Springer-Verlag, Berlin, Heidelberg, 1985) pp. 1- 76.

20. A. Wawrzyńczyk, Group Representations and Special Functions, (D. Reidel Publishing Company, PWN, Warszawa, 1984) pp. 310,311.

21 K.B. Wolf, Integral transform representations of $\mathrm{SL}(2, \Re)$, in Group Theoretical Methods in Physics, Proceedings, Cocoyoc, México 1980, ed. K.B. Wolf (Springer-Verlag, Berlin, Heidelberg, 1980) pp. 526-531. 